\title{
An Advanced In Vitro Model to Assess Glaucoma Onset
}

\author{
Sergio C. Saccàl , Sara Tirendi2,3, Sonia Scarfì 4 , Mario Passalacqua², Francesco Oddone 5 , \\ Carlo E. Traverso 1,6, Stefania Vernazza2,5\# and Anna M. Bassi2,3\# \\ ${ }^{1}$ IRCCS, San Martino General Hospital, Ophthalmology Unit, Genoa, Italy; ${ }^{2}$ Department of Experimental Medicine (DIMES), University of Genoa, \\ Genoa, Italy; ${ }^{3}$ Inter-University Center for the Promotion of the 3Rs Principles in Teaching \& Research (Centro 3R), Italy; ${ }^{4}$ Department of Earth, \\ Environmental and Life Sciences (DISTAV), University of Genoa, Genoa, Italy; ${ }^{5}$ IRCCS, Fondazione G.B. Bietti, Rome, Italy; ${ }^{6}$ Eye Clinic of Genoa, \\ San Martino General Hospital, Department of Neuroscience, Rehabilitation, Ophthalmology, Genetics, Maternal and Child Health (DiNOGMI), \\ University of Genoa, Genoa, Italy
}

\begin{abstract}
Glaucoma is the second leading cause of blindness worldwide. Currently, glaucoma treatments aim to lower intraocular pressure by decreasing aqueous humor production or increasing aqueous humor ouffow through pharmacological approaches or trabeculectomy. The lack of an effective cure requires new therapeutic strategies. We compared the biological responses of a three-dimensional trabecular meshwork model with or without perfusion bioreactor technology to better understand the early molecular changes induced by prolonged oxidative stress conditions induced by repeated daily peroxide exposure. We used standard 3D cultures of trabecular meshwork cells in Matrigel cultured under either static and dynamic conditions for one week. We studied changes in F-actin expression and organization in the cells, cellular metabolic activity, proinflammatory gene expression, expression of pro- and anti-apoptotic proteins, PARP-1 cleavage, and NFKB activation in the model. We demonstrate that the dynamic conditions improve the adaptive behavior of $3 \mathrm{D}$ trabecular meshwork cultures to chronic oxidative stress via offsetting pathway activation.
\end{abstract}

\section{Introduction}

Glaucoma is a neurodegenerative disease that affects $3.54 \%$ of the population aged $40-80$ years (Tham et al., 2014). There are several variants of this eye disease including primary open angle glaucoma (POAG), primary angle closure glaucoma, secondary glaucoma, and developmental glaucoma. Whereas in POAG, a high-tension form of glaucoma, there is an increase in the intraocular pressure (IOP), IOP is no longer considered the sole factor indicating the worsening of glaucoma in other forms of the disease, because glaucoma onset and progression can also occur within the normal IOP range (Kim and Park, 2019). Indeed, retinal ganglion cell (RGC) death depends on a wide range of molecular events that underlie the pathogenesis of glaucoma, and it is therefore crucial to further investigate the molecular mechanisms involved in RGC apoptosis in order to counteract this process.

Oxidative stress (OS) is regarded to be the cause of pathological outcomes including ischemic, oxidative, and inflammatory events that underlie glaucoma pathogenesis. The most common cause of the high-tension glaucoma cascade starts with OS at the trabecular meshwork (TM), which is the first element of the conventional outflow pathway (Saccà et al., 2016b). In fact, since the TM is the tissue most sensitive to OS, i.e., damage caused in the anterior chamber of the eye (Izzotti et al., 2009), many different outcomes, such as TM mitochondrial dysfunction, inflammatory cytokine release, impairment of extracellular matrix (ECM) components and their turnover, cellular senescence promotion and a consequent loss of cellularity, and others, ensue (Zhao et al., 2016; Kim and Kim, 2018). The resulting endothelial dysfunction, which affects the endothelial cells of the trabecular meshwork occurs in both normal-tension glaucoma and high-tension glaucoma (Saccà et al., 2019). Although the mechanisms by which the apoptotic signals develop towards RGCs are not yet known, it has been hypothesized that the glaucomatous effects on TM cells, with or without an increase of IOP, affect their gene and protein expression, generating molecular sig-

\footnotetext{
\# contributed equally
} 
nals, which, on reaching the head of the optic nerve, contribute to RGC death (Saccà et al., 2016a).

Most of the studies carried out this far to mimic different glaucomatous situations have used a variety of animal models (i.e., mouse, rat, rabbit, pig, cat, dog and monkey models). However, the obvious anatomical and morphological differences between animal and human eyes, together with the inflammation pathway triggered in the experimental approaches used to induce prolonged or transient elevation of intraocular pressure in animals, indicate that instead human-specific models of glaucoma are highly desirable (Bouhenni et al., 2012; Langley et al., 2017).

There is a growing interest in reliable 3D TM models as platforms to test new therapeutics because, unlike 2D cell cultures, they mimic the true nature of TM tissue in terms of morphology and environment (Brancato et al., 2018; Vernazza et al., 2019). In this regard, ex vivo models arising both from human and animal sources are the most widely used (Gonzalez et al., 2013; Li et al., 2019). However, the scarce availability of primary tissue limits their use in perfusion studies and drug testing (Waduthanthri et al., 2019). Therefore, 3D in vitro TM models could represent a good starting point to study several molecular features of glaucoma.

Recently, natural polymer scaffolds such as collagen-chondroitin sulfate and matrix-based Matrigel ${ }^{\circledR}$ have been used to study 3D-TM cell behavior under physiological and stress conditions and to mimic in vitro 3D-TM organization (Bouchemi et al., 2017; Osmond et al., 2017; Vernazza et al., 2019). Given its biological composition based on basement membrane components (i.e., laminin, collagen IV, heparin sulfate proteoglycans and entactin), matrix degrading enzymes, their inhibitors, and numerous growth factors, Matrige ${ }^{\circledR}$ has been used to perform the endothelial cell tube assay, grow 3D cancer cultures, perform the invasion assay, and to support organoid assembly and the expansion of undifferentiated human embryonic stem cells (Kohen et al., 2009; Hughes et al., 2010; Benton et al., 2014).

The aim of the present study was to define a more realistic in vitro model to study glaucoma onset and its outcomes by improving standard 3D-culture performance. For this purpose, we applied millifluidic bioreactor technology to 3D human trabecular meshwork cell (HTMC) in vitro models with the aim of allowing a continuous medium supply at a constant flow rate without exposing the cells to a high shear force (Giusti et al., 2014; Berger et al., 2018). We then analyzed the proinflammatory and proapoptotic effects of repeated administration of hydrogen peroxide in 3D-HTMC embedded in Matrigel ${ }^{\circledR}$ cultured under either static or dynamic conditions for one week.

\section{Materials and methods}

\section{Cell culture}

HTMC from normal healthy human adult eyes and Trabecular Meshwork Growth Medium (TMGM) were acquired from Cell Application Inc. (San Diego, CA, USA). Cell Application

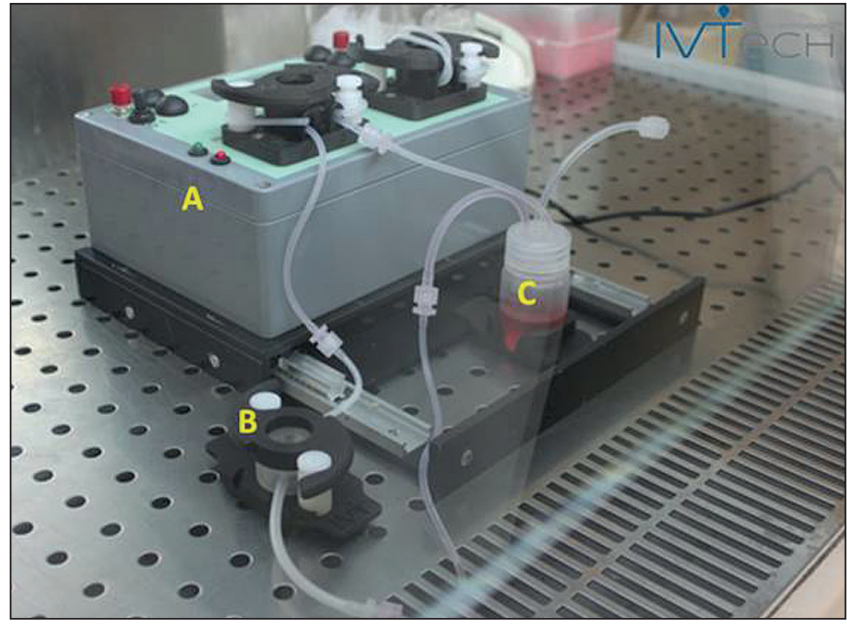

Fig. 1: Perfusion bioreactor circuit diagram

From the mixing bottle $(C)$, the medium is pumped by the action of the peristaltic pump (A), through the perfusion chamber where 3D-HTMCs were seeded (B), then it returns to the medium reservoir, completing the circuit. The image represents only one circuit, but the flow system includes two head pumps connected with at least four perfusion chambers (kindly provided by IVTech srl).

Inc. laboratory provided an official report of evidence that the HTMC cells express several markers related specifically to a trabecular phenotype and respond to dexamethasone treatment by increasing protein level expression of fibronectin, $\alpha$-smooth muscle actin, myocilin, and the cross-linked actin networks (CLAN) ${ }^{1}$. HTMCs were cultured according to consensus recommendations reported by Keller et al. (2018) and were maintained at $37^{\circ} \mathrm{C}$ in a humidified atmosphere containing $5 \% \mathrm{CO}_{2}$. All cell cultures were used at passages 2 to 6 and were found to be mycoplasma-free during regular checks with the Reagent Set Mycoplasma Euroclone (Euroclone ${ }^{\circledR}$ Milan, Italy).

The 3D-cultures were set up as previously described ${ }^{2}$ by embedding HTMC in $100 \%$ Corning $^{\circledR}$ Matrigel ${ }^{\circledR}$ Matrix (Corning Life Sciences, Tewksbury, MA USA) at a density of $2 \times 10^{6} / \mathrm{cm}^{3}$ seeded directly into a LiveBox 1 (IVTech S.r.l. - Massarosa, Italy) bioreactor culture chamber, $1.7 \mathrm{~cm}^{2}$ growth area/dish, and culturing them in either static or flow conditions (Vernazza et al., 2019).

\section{Dynamic 3D-HTMC system}

To study 3D-HTMC behavior under dynamic conditions, we used a sophisticated model of milli-scaled multi-organ devices in a single flow configuration (LB1, IVTech srl) (Ucciferri et al., 2014). The device is composed of a peristaltic pump (LF, IVTech srl), transparent culture chambers, and a mixing bottle, all equipped with inlet and outlet pipes on the sides that allow them to be interconnected. After seeding, each culture chamber containing the embedded cells was filled with $1 \mathrm{~mL}$ culture medium and placed in an incubator for $24 \mathrm{~h}$. The next day, the mixing

\footnotetext{
1 https://www.cellapplications.com/human-trabecular-meshwork-cells-htmc

2 https://doi.org/10.17504/protocols.io.574g9qw
} 
bottle was filled with $9 \mathrm{~mL}$ culture medium and the circuit was filled using the peristaltic pump. The culture medium circulated in the closed circuit at a constant flow rate of $70 \mu \mathrm{L} / \mathrm{min}$ with basal perfusion. The flow rate was chosen to overcome diffusional limitations and to avoid Matrige ${ }^{\circledR}$ degradation over time (Fig. 1 ). The medium was then replaced every 72 hours during the experimental time of 7 days.

\section{Chronic stress condition}

Prolonged oxidative stress was induced in both 3D-models over the course of a week by adding $500 \mu \mathrm{M} \mathrm{H}_{2} \mathrm{O}_{2}$ to each well once a day for $2 \mathrm{~h}$, followed by $22 \mathrm{~h}$ of recovery, according to Poehlmann et al. (2013) and Kaczara et al. (2010). All molecular analyses on static and dynamic 3D-HTMC cultures were conducted once cells were freed from Corning ${ }^{\circledR}$ Matrige $l^{\circledR}$ Matrix (Corning Life Sciences, Tewksbury, MA USA) by Corning Cell Recovery (Corning Life Sciences), according to the manufacturer's instructions.

\section{Confocal analysis}

At each selected time point, the 3D-HTMCs, cultured and treated as mentioned above, were set in $4 \%$ paraformaldehyde and permeabilized with $0.3 \%$ Triton X-100 (Sigma Aldrich ${ }^{\circledR}$, Milan, Italy). Nuclei were stained with To-Pro ${ }^{\text {TM}}$-3 Iodide 642/641 (Thermofisher Scientific Inc., Monza, Italy) and the actin cytoskeleton was visualized using Phalloidin Alexa Fluor 488 (Cell Signaling Technology, Danvers, USA). Fluorescence signals were captured at $60 \mathrm{X}$ magnification, by Leica TSC SP microscope (Leica Microsystem, Wetzlar, Germany). Both fluorescence signals and F-actin intensity were quantified by FijiImageJ software, an open-source platform for biological image analysis. Signals from different fluorescent probes were taken in sequential scan settings (3D reconstruction images).

\section{Alamar Blue assay}

The metabolic activity of the 3D-HTMCs was assessed daily using the $\mathrm{AB}$ assay (Invitrogen ${ }^{\mathrm{TM}}$, Thermo Fisher Scientific Inc.,
Monza, Italy) within the last $4 \mathrm{~h}$ of the $22 \mathrm{~h}$ recovery time, during which medium circulation was stopped, according to the manufacturer's instructions. In short, $10 \%$ (v/v) AB solution was added to each well and, after $4 \mathrm{~h}$ of incubation, resazurin reduction was quantified spectrophotometrically at wavelengths of 570 and $630 \mathrm{~nm}$. The results were expressed as fold reduction activity of treated vs untreated 3D-HTMCs.

RNA extraction, $c D N A$ synthesis and $q P C R$ analyses

3D-HTMCs $\left(5 \times 10^{5}\right)$ were treated as described above. A gene expression profile was obtained at $48 \mathrm{~h}$ by qPCR analysis and compared to control cells. Total RNA was extracted using the RNeasy Micro Kit (Qiagen, Milan, Italy) according to the manufacturer's instructions. A NanoDrop spectrophotometer (Nanodrop Technologies, Wilmington, DE, USA) was used to quantify the RNA. cDNA was synthesized using SuperScript ${ }^{\mathrm{TM}}$ III First Strand Synthesis System (ThermoFisher Scientific). Primers (Tab. 1) were designed using the Beacon Designer 7.0 software (Premier Biosoft International, Palo Alto CA, USA) and obtained from TibMolBiol (Genova, Italy). PCR reactions were performed as described elsewhere (Vernazza et al., 2019). Values were normalized to ubiquitin (reference gene) mRNA expression. Data was analyzed using the DNA Engine Opticon ${ }^{\circledR}$ 3 Real-Time Detection System Software program (3.03 version) and, in order to calculate the relative gene expression compared to an untreated (control) calibrator sample, the comparative threshold Ct method (Aarskog and Vedeler, 2000) was used within the Gene Expression Analysis for iCycler iQ Real Time Detection System software (Bio-Rad) (Vandesompele et al., 2002).

\section{Human apoptosis array}

Apoptosis was investigated by the semi-quantitative detection of 43 human apoptotic proteins on a customized Human Apoptosis Array C1 chip (RayBio ${ }^{\circledR}$; Norcross, GA) (Tab. 2), according to the manufacturer's instructions. The intensity of the protein array signals was analyzed using a BIORAD Geldoc 2000, and each

Tab. 1: Primer sequences used for real time quantitative polymerase chain reaction analysis

\begin{tabular}{|l|l|l|l|}
\hline Gene & GenBank & Forward & Reverse \\
\hline IL-1a & NM_000575.4 & CAATCTGTGTCTCTGAGTATC & TCAACCGTCTCTTCTTCA \\
\hline IL-1 $\beta$ & NM_000576.2 & TGATGGCTTATTACAGTGGCAATG & GTAGTGGTGGTCGGAGATTCG \\
\hline IL-6 & NM_001318095.1 & CAGATTTGAGAGTAGTGAGGAAC & CGCAGAATGAGATGAGTTGTC \\
\hline MMP-1 & NM_001145938.1 & GGTGATGAAGCAGCCCAGATG & CAGAGGTGTGACATTACTCCAGAG \\
\hline MMP-3 & NM_002422.5 & TAATAATTCTTCACCTAAGTCTCT & AGATTCACGCTCAAGTTC \\
\hline MMP-9 & NM_004994.2 & AACCAATCTCACCGACAGG & CGACTCTCCACGCATCTC \\
\hline TNFa & NM_000594.4 & GTGAGGAGGACGAACATC & GAGCCAGAAGAGGTTGAG \\
\hline TGF- $\beta 2$ & NM_001135599.3 & AACCTCTAACCATTCTCTACTACA & CGTCGTCATCATCATTATCATCA \\
\hline Ubiquitin C & NM_021009.7 & ATTTGGGTCGCAGTTCTTG & TGCCTTGACATTCTCGATGGT \\
\hline HPRT1 & NM_000194.3 & GGTCAGGCAGTATAATCCAAAG & TTCATTATAGTCAAGGGCATATCC \\
\hline
\end{tabular}


Tab. 2: The mini map of Human Apoptosis Array C1 (according to RayBio ${ }^{\circledR}$ manufacturer manual)

\begin{tabular}{|c|c|c|c|c|c|c|c|c|c|c|c|c|c|c|}
\hline & A & B & C & D & $E$ & $F$ & $G$ & H & 1 & $J$ & K & L & M & $\mathrm{N}$ \\
\hline$\frac{1}{2}$ & POS & POS & NEG & NEG & Blank & Blank & bad & bax & Bcl2 & Bcl2-w & BID & BIM & Caspase3 & Caspase8 \\
\hline$\frac{3}{4}$ & CD40 & CD40L & CIAP2 & CytoC & DR6 & Fas & FasL & Blank & Hsp27 & Hsp60 & Hsp70 & HTRA2 & IGF1 & IGF2 \\
\hline 6 & IGFBP1 & IGFBP2 & IGFBP3 & IGFBP4 & IGFBP5 & IGFBP6 & IGF-1R & Livin & P21 & P27 & P53 & SMAC & Survivn & TNF RI \\
\hline$\frac{7}{8}$ & TNF RII & TNFa & TNF $\beta$ & \begin{tabular}{|l} 
TRAIL \\
R1
\end{tabular} & $\begin{array}{l}\text { TRAIL } \\
\text { R2 }\end{array}$ & $\begin{array}{l}\text { TRAIL } \\
\text { R3 }\end{array}$ & \begin{tabular}{|l} 
TRAIL \\
R4
\end{tabular} & XIAP & Blank & Blank & NEG & NEG & POS & POS \\
\hline
\end{tabular}

(A)

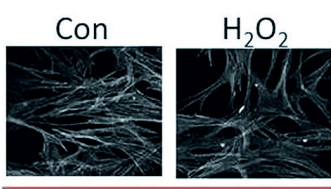

Static model

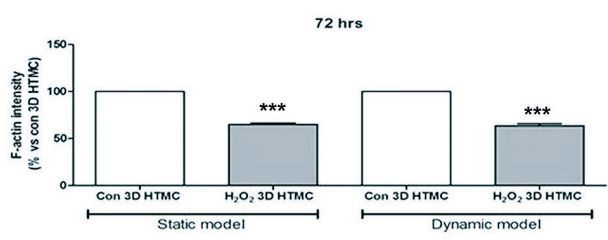

(B)
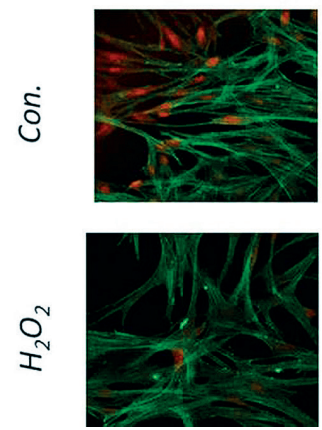

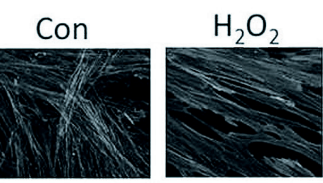

Dynamic model
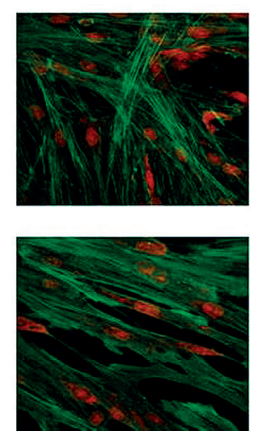

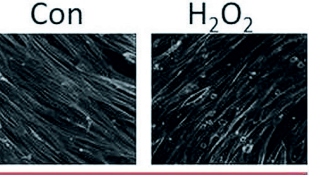

Static model
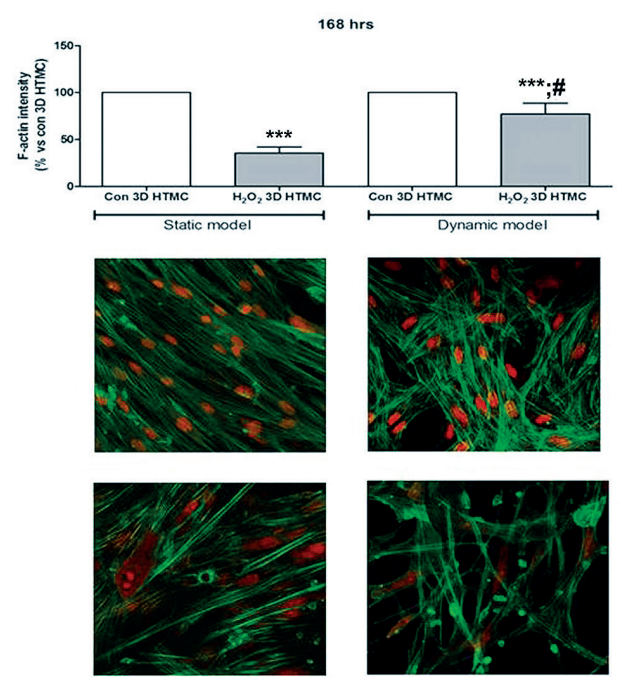
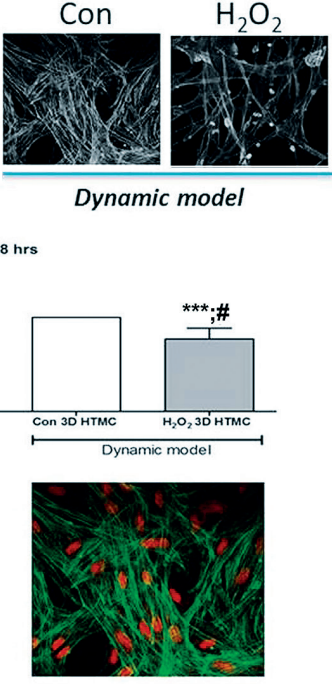

Dynamic model

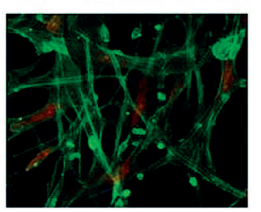

Fig. 2: F-actin intensity measurement and confocal analysis

(A) F-actin intensity was calculated in at least three images of the same condition. Each measurement included ten areas of interest per image. Data represent the mean $\pm S D$ of 6 independent experiments. (B) Confocal microscopy analyses of nucleus and cytoskeletal markers were performed on untreated (con) and $\mathrm{H}_{2} \mathrm{O}_{2}-3 \mathrm{D}-\mathrm{HTMCs}$ in static and in dynamic conditions after 72 and $168 \mathrm{~h}$. Representative images are related to immunoreactivity for To- Pro $^{\mathrm{TM}}$ and Phalloidin, as nuclear and cytoskeleton markers, respectively. Merged images show cytoskeleton plus nucleus. Fluorescence signals were captured at $60 x$ magnification. ${ }^{* \star *} \mathrm{p}<0.0001$ vs. untreated (con) $3 D$ HTMCs and \#p $<0.05$ dynamic $\mathrm{H}_{2} \mathrm{O}_{2} 3 \mathrm{D}-\mathrm{HTMC}$ vs static $\mathrm{H}_{2} \mathrm{O}_{2}$ 3D-HTMC (one-way ANOVA followed by Bonferroni's posttest).

protein spot was normalized against positive control spots printed on each membrane. The data analysis was conducted according to the protocol directions of Human Apoptosis Array C1, and the relative protein expression on different arrays was extrapolated by using the algorithm, according to the Human Apoptosis Array C1 protocol.

\section{Western blotting}

Total proteins were extracted from HTMCs as described elsewhere (Vernazza et al., 2019) and were resolved in Any $\mathrm{kD}^{\mathrm{TM}}$ mini precast gel (Bio-Rad Laboratories, Inc., Hercules, CA, USA) in SDS-PAGE running buffer. Blots were incubated with antibodies to PARP1, phospho-NF-кB p65 and Ser 536 (Cell Sig- 
naling Technology, Danvers, MA, USA). To normalize loading of the lanes, blots were stripped and incubated with anti-GAP$\mathrm{DH}$ antibody. The proteins were detected by Western Bright ${ }^{\mathrm{TM}}$ ECL (Advansta, CA, USA), exposed to film and analyzed using a BIORAD Geldoc 2000. Densitometrical data obtained from Quantity One software (Bio-Rad Laboratories, Inc., Hercules, CA, USA) were subjected to statistical analysis and normalized against the housekeeping GAPDH. The results were expressed as fold versus untreated cultures, respectively.

\section{Statistical analysis}

Reported data are expressed as mean $\pm \mathrm{SD}$ and results were analyzed using two-way analysis of variance (ANOVA) for single comparison or two-way ANOVA followed by Bonferroni's posttest for multiple comparisons. GraphPad Prism for Windows (version 5.03, GraphPad Software, Inc., La Jolla, CA, USA) was used. Statistically significant differences were set at $*, \mathrm{p}<0.05$; $* *, \mathrm{p}<0.01 ; * * *, \mathrm{p}<0.001$.

\section{Results}

\subsection{Confocal analysis}

To understand the dynamics of F-actin in 3D-HTMC under both static and dynamic conditions during repeated 2 hour-exposures to peroxide, the F-actin intensity associated with the cytoskeleton was quantified (Fig. 2, panel A). At $72 \mathrm{~h}$, a reduction by about $40 \%$ in F-actin intensity was observed in both culture models compared to that of untreated 3D-HTMCs. However, at $168 \mathrm{~h}, \mathrm{~F}$-actin intensity had mostly recovered only in the treated dynamic 3D-HTMC model, while in the static 3D-HTMC model F-actin intensity was further reduced by about $64 \%$ and $42 \%$, compared to untreated static 3D-HTMC and treated dynamic 3D-HTMC, respectively.

Moreover, the spatial organization of 3D-HTMCs cultures at 72 and $168 \mathrm{~h}$ was analyzed by confocal microscopy (Fig. 2, panel B). In 3D-static HTMC cultures, the actin microfilaments, detected by fluorescent probe FITC-Phallodin were uniformly distributed in parallel lines along the longitudinal axis and much cell-to-cell interaction was detected. However, in dynamic 3DHTMCs, actin microfilaments showed a less orderly distribution of the cells embedded in the matrix. In both culture conditions, the $\mathrm{H}_{2} \mathrm{O}_{2}$-treated 3D-HTMCs showed an increase of nuclear size, labeled by fluorescent probe To-Pro ${ }^{\mathrm{TM}}$, while actin microfilaments were thicker, tense and distributed in radial manner only in dynamic conditions.

\subsection{Alamar Blue assay}

The effects of chronic $500 \mu \mathrm{M} \mathrm{H}_{2} \mathrm{O}_{2}$ exposure were measured every $24 \mathrm{~h}$ up to $168 \mathrm{~h}$ by Alamar Blue assay in 3D-HTMC cultured in static and dynamic conditions (Fig. 3).

Over the first $24 \mathrm{~h}$, the metabolic activity of the 3D-static HTMC cultures showed a significant increase compared to the untreated cultures (by about 50\%). The levels decreased time-dependently at the later exposure times and returned to levels of untreated cultures at $96 \mathrm{~h}$. At later time points, metabolic activity dropped significantly below that of untreated cultures.

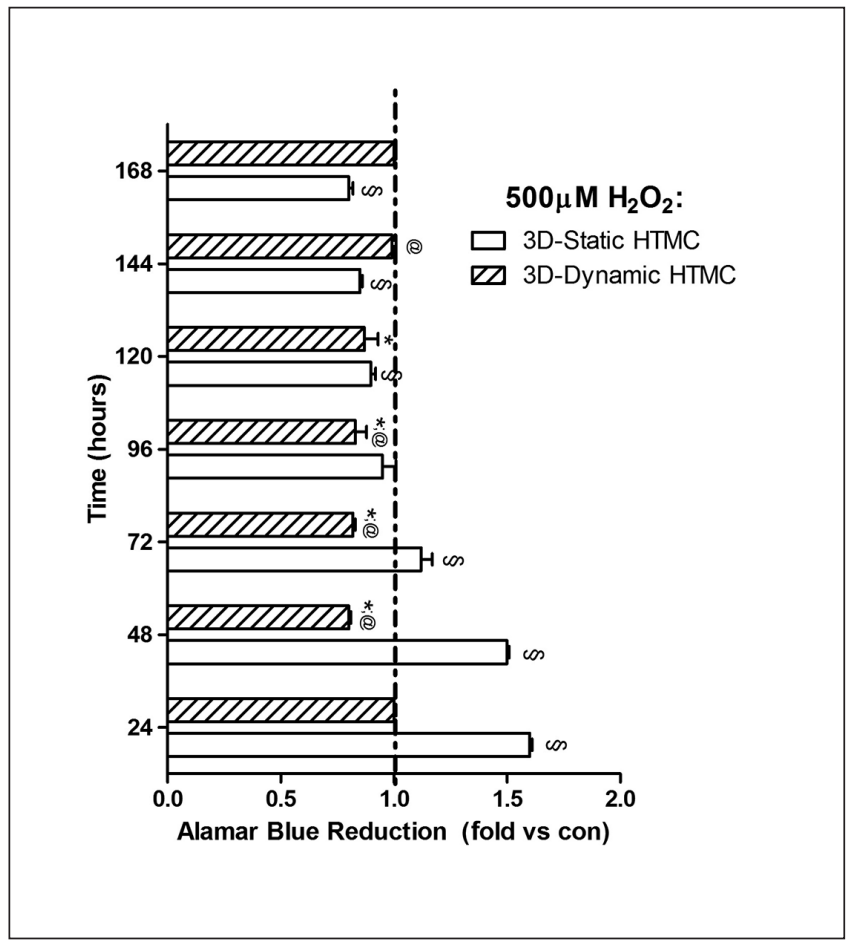

Fig. 3: Metabolic activity of 3D-HTMCs cultured in static or dynamic conditions and subjected to repeated daily $500 \mu \mathrm{M}$ $\mathrm{H}_{2} \mathrm{O}_{2}$ treatment

Metabolic state of 3D HTMCs cultured in static and dynamic conditions was analyzed by Alamar blue assay during the last $4 \mathrm{~h}$ of the $22 \mathrm{~h}$ recovery time after daily exposure to peroxide. The black dotted line represents the respective metabolic activity of untreated (con) 3D HTMCs cultured in static or dynamic conditions; the white bars represent the 3D HTMCs treated with $500 \mu \mathrm{M} \mathrm{H}_{2} \mathrm{O}_{2}$. Data are expressed as fold vs untreated 3D-HTMC, and each value represents the mean $\pm S D$ of 3 independent experiments running in triplicate. ${ }^{*}, \mathrm{p} p<0.001$ vs respective untreated (con) 3D-HTMCs; @p $<0.001$ vs treated 3D-static HTMC (two-way ANOVA followed by Bonferroni posttests).

In contrast, the metabolic activity of 3D-dynamic HTMCs remained comparable to that of untreated cultures over the first 24 hours. Repeated OS exposure then resulted in a significant decrease in the metabolic activity (by about $20 \%$ vs untreated dynamic cultures), which recovered slowly to return to levels comparable with untreated cultures at $144 \mathrm{~h}$ and $168 \mathrm{~h}$.

\section{3 qPCR}

In order to compare cytokine production, MMP regulation, and ECM gene expression after OS treatment on 3D-HTMCs cultured in static vs dynamic conditions, the cells were treated as described above for $48 \mathrm{~h}$. Gene expression levels of IL-1 $\alpha$, IL-1 $\beta$, IL6, TNF $\alpha$, TGF $\beta$, MMP1, MMP3, MMP9, COL1A1 and FN1 were analyzed by qPCR (Fig. 4).

OS-treated 3D-dynamic HTMCs showed an up-regulation of IL-1 $\beta$, TGF $\beta$, MMP1, MMP3 and MMP9 compared both to untreated cultures and OS-treated 3D-static HTMCs. OS-treat- 
(A)

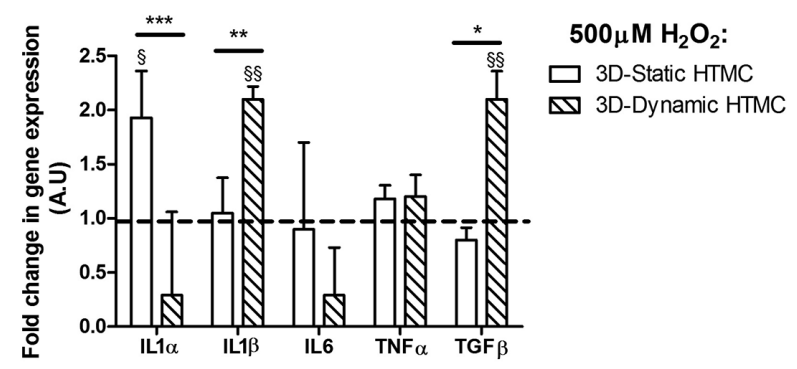

(B)

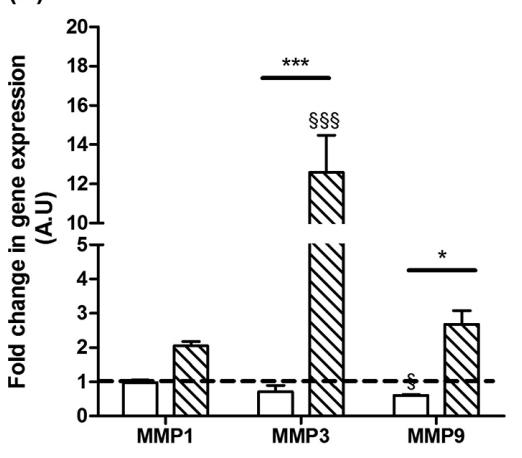

(C)

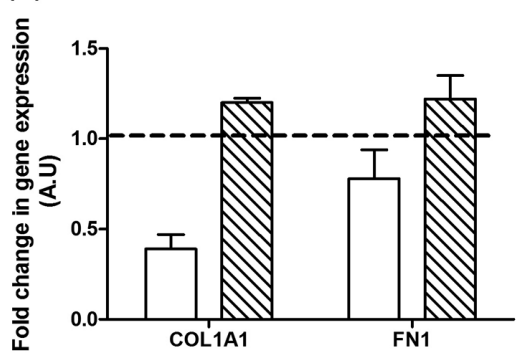

ed 3D-static HTMCs evidenced a significant increase only for IL-1 $\alpha$ levels compared both to untreated cultures and OS-treated 3D-dynamic HTMCs.

\subsection{Human apoptosis array}

After prolonged OS, multiple markers of the apoptosis pathway were analyzed on both 3D-HTMC models using a microarray for 43 pro- or anti-apoptotic proteins. In Figure 5, we report only the levels of those proteins that were significantly modulated in one or in both 3D-HTMC models.

At the $48 \mathrm{~h}$ timepoint, 3D-static HTMCs evidenced a significant increase of pro-apoptotic BID, BIM, Caspase 3, p53, Smac, TNF-R1 proteins. This modulation was even stronger at $72 \mathrm{~h}$ for all above proteins, except for proapoptotic p53 and Smac. Regarding antiapoptotic molecules, there was a significant increase in Survivin, IGFBP1 and IGF1 at the $48 \mathrm{~h}$ timepoint, and a stronger increase in BCL2, BCLw, survivin, x IAP, CD 40 and IGF1 at $72 \mathrm{~h}$. These modulations of pro- and anti-apoptotic markers had all returned to baseline in 3D-static cultures at 168 hours,

In dynamic conditions, at the $48 \mathrm{~h}$ timepoint, pro-apoptotic BAD, BID, BAX, BIM, CytoC, Smac and TNF $\alpha$, TNF $\alpha / \mathrm{TN}-$ FR1, TNF $\alpha /$ TNFR2 protein levels were significantly increased compared to those of control cultures. At $72 \mathrm{~h}$, the the levels of almost all markers had returned to baseline levels, except for TNF $\alpha$, TNF $\alpha /$ TNFR1, TNF $\alpha /$ TNFR2 which were even higher at $168 \mathrm{~h}$. As for anti-apoptotic patterns, only IGFBP1 evidenced a significant increase at $48 \mathrm{~h}$, while BCL2, BCLw, Hsp70, CD40, IGFBP1 and IGF1 were increased up to 3 -fold at $168 \mathrm{~h}$.

\subsection{Western blot analysis}

Analysis of PARP-1 cleavage was performed on 3D-HTMCs after $168 \mathrm{~h}$ chronic exposure to peroxide (Fig. 6). A marked and significant increase of PARP-1 cleavage levels was detected only in 3D-static HTMCs cultures, while in 3D-dynamic HTMCs no PARP-1 cleavage was detectable (Panel A).

NF- $\kappa B$ transactivation, as an inflammatory/anti-apoptotic response marker, was analyzed in terms of the ratio between the levels of phospho-NF- $\mathrm{kB} \mathrm{p65}$, the activated form of NF- $\mathrm{kB}$, versus total NF- $\mathrm{KB}$ (Panel B). A remarkable NF- $\mathrm{KB}$ activation occurred only in 3D-HTMC cultured in dynamic conditions.

\section{Discussion}

The aim of the study was to develop a relevant $3 \mathrm{D}$ in vitro model of HTMC using Matrigel ${ }^{\circledR}$ and bioreactor technologies to explore, in a more physiological way, the first molecular changes in the human trabecular meshwork during prolonged oxidative stress.

There are several inducible glaucoma animal models that allow evaluation of ganglion cell axon damage through the experimental increase of IOP or direct damage of the optic nerve (Burgoyne, 2015; Ishikawa et al., 2015; Struebing and Geisert, 2015; Evangelho et al., 2019). However, these approaches explain only part of the molecular mechanisms of glaucoma and, until today, the only clinically modifiable risk factor remains IOP. Species-specific differences may lead to overestimation of 


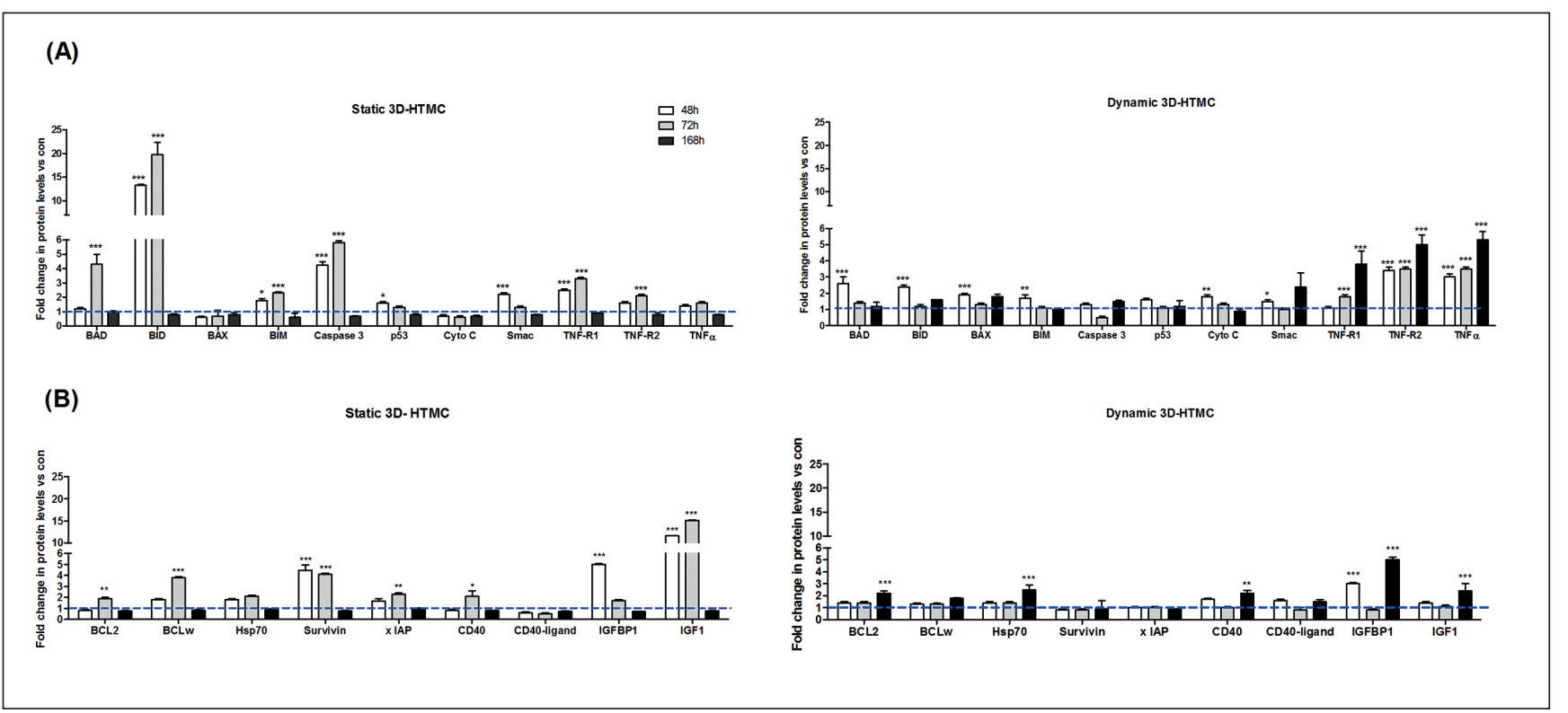

Fig. 5: Expression of pro- and anti-apoptotic proteins at 48, 72 and $168 \mathrm{~h}$ in $3 \mathrm{D}-\mathrm{HTMCs}$ cultured in static or dynamic conditions and subjected to repeated daily $500 \mu \mathrm{M} \mathrm{H}_{2} \mathrm{O}_{2}$ treatment

Analysis of pro- and anti-apoptotic protein levels (Panel A and Panel B, respectively) in 3D-HTMCs cultured in static and dynamic conditions were performed after 48, 72 and $168 \mathrm{~h}$ by Human Antibody Array C1 (RayBio ${ }^{\circledR} \mathrm{C}$-series). Only significantly modulated markers are reported in the graphs. The light blue dotted line represents the protein level of untreated HTMC for each protein examined. Twelve individual models were arrayed (six static 3D-HTMCs plus six dynamic 3D-HTMC) and per experiment the intensity of the Positive Control Spot was used to normalize signal responses for comparison of results across multiple arrays. ${ }^{*} p<0.05 ;{ }^{* *} p<0.01 ;{ }^{* * *} p<0.001$ vs respective untreated cultures (one-way ANOVA).

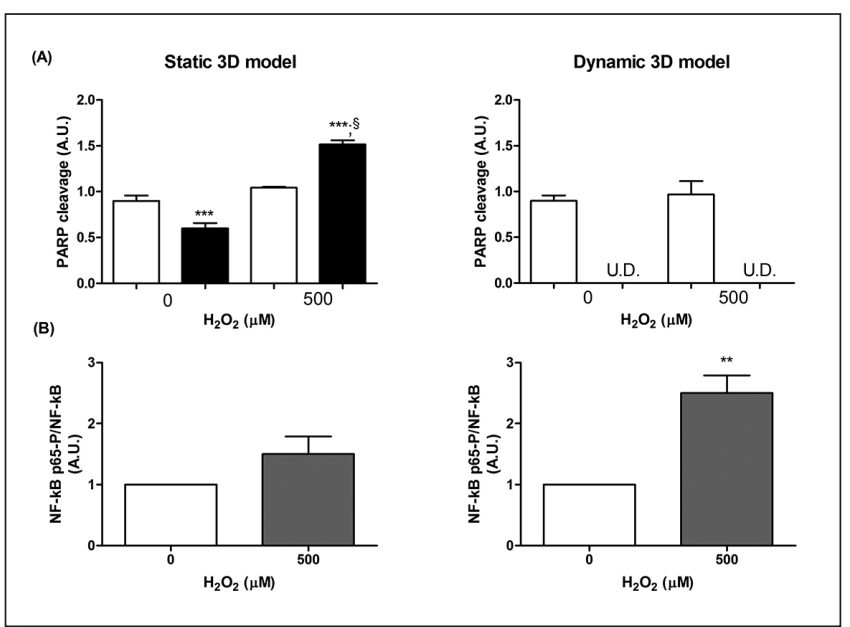

Fig. 6: PARP1 cleavage levels and NF-KB levels at $168 \mathrm{~h}$ in 3D-HTMCs cultured in static or dynamic conditions and subjected to repeated daily $500 \mu \mathrm{M} \mathrm{H}_{2} \mathrm{O}_{2}$ treatment The analysis was performed on 3D static (left panel) and 3D dynamic (right panel) cultures by immunoblotting. Bars represent the ratio of cleaved PARP1 and phosphoNF-kBp65/NF-kB, and are expressed as arbitrary units (AU). Data represent the mean \pm SD of 2 independent experiments, running in triplicate.

${ }^{* \star *} p<0.0001 ;{ }^{* \star} p<0.01$ vs untreated $3 D$ cultures and $\$ p<0.0001$ 3D-HTMC treated vs 3D-HTMC control cultures (two-way ANOVA). effects or oversight of fundamental issues, and therefore results from animal experiments cannot always be translated to humans. There is therefore a pressing need for translationally-relevant human biology-based advanced models to help us better understand the disease in humans at multiple biological scales. This information then may be arranged into adverse outcome pathway constructs.

In the last few years, 3D-cultures together with 3D-culture techniques have allowed the development of increasingly physiologically relevant species-specific in vitro models of diseases. In particular, the development of techniques allowing perfusion flows ranging from milli- to micro-fluidics has enhanced the performance of 3D-cultures. Indeed, Ucciferri et al. (2014) and Ahluwalia (2017) reported that cells and tissues cultured in vitro under specific conditions maintain power law metabolic scaling, confirming the physiological relevance of these downscaled in vitro systems.

In the small dimension bioreactor scenario, cell-culture chambers only a few milliliters in volume (i.e., "milli-fluidic" chambers) simulate physiological environmental conditions, exposing cells to a flow that is comparable with the human circulation (Giusti et al., 2014), using a peristaltic pump. In this way, cells can interact with each other in a physiological manner.

Previous studies (Bouchemi et al., 2017; Vernazza et al., 2019) have shown that 3D-HTMC cultures are more physiological models than 2D-cultures. To further improve our 3D-HTMC cul- 
ture, we applied dynamic flow using the millifluidic technique and compared the biological responses of the cells cultured under dynamic conditions to those of cells cultured under static conditions to repeated oxidative stress, which is closely linked both to TM pathological changes and glaucoma (Saccà and Izzotti, 2014; Zhao et al., 2016).

The emergence of an aqueous humor outflow resistance is ascribed to cytoskeletal reorganization, and changes in cell shape, contractile properties, and cell-to-cell/ECM attachments (Xiang et al., 2010). Thus, we analyzed changes to 3D-HTMC morphology by confocal microscopy after repeated exposures to peroxide (Fig. 2). F-actin was chosen as cytoskeleton marker because it is abundant in TM cells. In our experimental conditions, at the 72 and $168 \mathrm{~h}$ timepoints, actin microfilaments appeared thinned and nuclei were enlarged in both static and dynamic 3D-HTMCs culture models compared to untreated cultures. These morphological changes confirmed that, besides biomechanical insults (Saccà et al., 2016b), prolonged OS can modify TM cell shape (i.e., F-actin cytoskeleton reorganization), likely through changes in gene expression.

Actin cytoskeleton depolymerization is one of the ROS mechanisms by which cell barrier functions may be impaired (Boardman et al., 2004). The reduction of F-actin intensity at $72 \mathrm{~h}$ showed the same trend for both the static and dynamic culture conditions. However, at $168 \mathrm{~h}$, only 3D-HTMC treated under dynamic conditions showed a partial recovery of F-actin intensity. This could be due to the better nutrient supply provided by the bioreactor system (Stapulionis et al., 1997).

Moreover, the expression of IL- $1 \alpha$, IL-1 $\beta$, IL- 6, TNF $\alpha$ and TGF $\beta$ was investigated by qPCR. At the $48 \mathrm{~h}$ time point, we found significant up-regulation of TGF- $\beta 1$ and IL- $1 \beta$, two crucial markers involved both in ECM remodeling (Pulliero et al., 2014; Lv et al., 2017) and in glaucoma acute inflammatory response (Taurone et al., 2015; Wang et al., 2017), but only in 3D-dynamic HTMC cultures.

The ECM changes in the TM play an important role in increasing both aqueous humor outflow resistance and IOP (Fig. 4, panel A). Indeed, several molecular factors interact with one another to promote ECM synthesis or its degradation, changing its basic properties (Fuchshofer and Tamm, 2009). Therefore, the expression of MMP, COL1A1 and FN1 was also evaluated. The results indicate that the peroxide exposure only changed the expression of cellular proteases in order to counteract OS-induced outflow resistance (Micheal et al., 2013; Singh et al., 2015) in 3D-dynamic HTMC. However, the expression of adhesion molecules did not show significant differences compared to the untreated 3D HTMC models, even though 3D-HTMC subjected to oxidative stress and cultured under dynamic conditions showed a significant COL1A1 up-regulation $(\mathrm{p}<0.01)$ compared to 3D-HTMC treated under static conditions. These results highlight that more physiological culture conditions are better able to mimic homeostatic TM cell responses found in vivo to adjust outflow resistance (Wang et al., 2001; Acott and Kelley, 2008). Thus, it is conceivable that our model mimics in vivo conditions found at early stages of glaucoma.

In this study we provide evidence that 3D-models, compared to $2 \mathrm{D}$ cultures commonly used for in vitro glaucoma studies, maintain tissue architecture, which represents an important hallmark for the tissue function maintenance found in vivo. However, a dynamic environment allows a better maintenance of cell structures than static culture conditions, also confirmed by confocal analysis, and this ability better supports cellular polarization, sustaining, as a consequence, long-term viability of the cells. Indeed, the modulation of apoptosis markers and NF- $\mathrm{\kappa B}$ protein levels in 3D-dynamic HTMC cultures showed a more efficient adaptive response over time to OS-damage, compared to 3D-static models (Zahir and Weaver, 2004), and triggered the inflammation cascade, as happens in vivo during glaucoma occurrence.

We also observed an increase in pro-apoptotic proteins including $\mathrm{BAD}, \mathrm{BIM}, \mathrm{BID}$, and cytochrome $\mathrm{C}$ in the $3 \mathrm{D}$-dynamic HTMCs only at the earliest timepoint of pro-oxidant stimulus exposure, and a gradual increase over time of TNF $\alpha$ and TNFR1. However, at $168 \mathrm{~h}$ a stronger increase in anti-apoptotic markers, including survivin and IGFPB1 and also the cell proliferation activator TNFR2 was found to counterbalance the apoptotic response (Fig. 5). These results, together with the uncleaved PARP1, the increase of phosphoNF- $\mathrm{kBp} 65$ rate compared to total NF-kB level, and the healthy metabolic state are in favor of cell survival rather than apoptosis (Fig. 6).

On the other hand, 3D-static HTMC cultures evidenced an overexpression of BAD, BID and Caspase 3 and, to a lesser extent, also of TNRF1, as well as a marked induction of anti-apoptotic proteins such as BCL2, BCLw, survivin, $\mathrm{x}$ IAP, CD 40 and IGF1 up to $72 \mathrm{~h}$ of OS-exposure. Nevertheless, after $168 \mathrm{~h}$ exposure to repeated sub-toxic stress, we reported PARP1 cleavage, no activation of NF- $\mathrm{KB}$ and a reduced metabolic activity leading to hypothesize that 3D-static HTMCs followed the apoptosis pathway (Fig. 3,6) (Elmore, 2007; Vanamee and Faustman, 2017).

In conclusion, dynamic 3D-HTMCs culture models allow for cellular function preservation over time. These features enable the cells to outlast prolonged stress attacks, and this is what is needed for a relevant in vitro model. Indeed, glaucoma is a chronic disease, which only shows effects a long time after the beginning of TM damage. This in vitro biodynamic platform can be further developed to become a useful tool to identify key events of damage onset and its long-term complications, such as blindness, by mimicking tissue-crosstalk with other tissues by joining different modules/chambers in series. In this way, it will be possible to analyze the stages of cell damage that underlie glaucoma and its adverse outcomes step-by-step.

\section{References}

Aarskog, N. and Vedeler, C. (2000). Real-time quantitative polymerase chain reaction. Hum Genet 107, 494-498. doi:10.1007/ s004390000399

Acott, T. S. and Kelley, M. J. (2008). Extracellular matrix in the trabecular meshwork. Exp Eye Res 86, 543-561. doi:10.1016/j. exer.2008.01.013

Ahluwalia, A. (2017). Allometric scaling in-vitro. Sci Rep 7, 42113. doi:10.1038/srep42113

Benton, G., Arnaoutova, I., George, J. et al. (2014). Matrigel: From discovery and ECM mimicry to assays and mod- 
els for cancer research. Adv Drug Deliv Rev 79-80, 3-18. doi:10.1016/j.addr.2014.06.005

Berger, E., Magliaro, C., Paczia, N. et al. (2018). Millifluidic culture improves human midbrain organoid vitality and differentiation. Lab Chip 18, 3172-3183. doi:10.1039/C8LC00206A

Boardman, K. C., Aryal, A. M., Miller, W. M. et al. (2004). Actin re-distribution in response to hydrogen peroxide in airway epithelial cells. J Cell Physiol 199, 57-66. doi:10.1002/jcp.10451

Bouchemi, M., Roubeix, C., Kessal, K. et al. (2017). Effect of benzalkonium chloride on trabecular meshwork cells in a new in vitro 3D trabecular meshwork model for glaucoma. Toxicol In Vitro 41, 21-29. doi:10.1016/j.tiv.2017.02.006

Bouhenni, R. A., Dunmire, J., Sewell, A. et al. (2012). Animal models of glaucoma. J Biomed Biotechnol 2012, 692609. doi:10.1155/2012/692609

Brancato, V., Gioiella, F., Imparato, G. et al. (2018). 3D breast cancer microtissue reveals the role of tumor microenvironment on the transport and efficacy of free-doxorubicin in vitro. Acta Biomater 75, 200-212. doi:10.1016/j.actbio.2018.05.055

Burgoyne, C. F. (2015). The non-human primate experimental glaucoma model. Exp Eye Res 141, 57-73. doi:10.1016/j. exer.2015.06.005

Elmore, S. (2007). Apoptosis: A review of programmed cell death. Toxicol Pathol 35, 495-516. doi:10.1080/01926230701320337

Evangelho, K., Mastronardi, C. A. and de-la-Torre, A. (2019). Experimental models of glaucoma: A powerful translational tool for the future development of new therapies for glaucoma in humans - A review of the literature. Medicina (Mex) 55, 280. doi:10.3390/medicina55060280

Fuchshofer, R. and Tamm, E. R. (2009). Modulation of extracellular matrix turnover in the trabecular meshwork. Exp Eye Res 88, 683-688. doi:10.1016/j.exer.2009.01.005

Giusti, S., Sbrana, T., La Marca, M. et al. (2014). A novel dual-flow bioreactor simulates increased fluorescein permeability in epithelial tissue barriers. Biotechnol J 9, 1175-1184. doi:10.1002/biot.201400004

Gonzalez, J. M., Hamm-Alvarez, S. and Tan, J. C. H. (2013). Analyzing live cellularity in the human trabecular meshwork. Invest Opthalmol Vis Sci 54, 1039-1047. doi:10.1167/ iovs.12-10479

Hughes, C. S., Postovit, L. M. and Lajoie, G. A. (2010). Matrigel: A complex protein mixture required for optimal growth of cell culture. Proteomics 10, 1886-1890. doi:10.1002/pmic. 200900758

Ishikawa, M., Yoshitomi, T., Zorumski, C. F. et al. (2015). Experimentally induced mammalian models of glaucoma. Biomed Res Int 2015, 281214. doi:10.1155/2015/281214

Izzotti, A., Saccà, S. C., Longobardi, M. et al. (2009). Sensitivity of ocular anterior chamber tissues to oxidative damage and its relevance to the pathogenesis of glaucoma. Invest Ophthalmol Vis Sci 50, 5251-5258. doi:10.1167/iovs.09-3871

Kaczara, P., Sarna, T. and Burke, J. M. (2010). Dynamics of $\mathrm{H}_{2} \mathrm{O}_{2}$ availability to ARPE-19 cultures in models of oxidative stress. Free Radic Biol Med 48, 1064-1070. doi:10.1016/j. freeradbiomed.2010.01.022

Keller, K. E., Bhattacharya, S. K., Borrás, T. et al. (2018). Consensus recommendations for trabecular meshwork cell isola- tion, characterization and culture. Exp Eye Res 171, 164-173. doi:10.1016/j.exer.2018.03.001

Kim, S. H. and Kim, H. (2018). Inhibitory effect of astaxanthin on oxidative stress-induced mitochondrial dysfunction - A mini-review. Nutrients 10, 1137. doi:10.3390/nu10091137

Kim, Y. W. and Park, K. H. (2019). Exogenous influences on intraocular pressure. Br J Ophthalmol 2018, 313381. doi:10.1136/bjophthalmol-2018-313381

Kohen, N. T., Little, L. E. and Healy, K. E. (2009). Characterization of Matrigel interfaces during defined human embryonic stem cell culture. Biointerphases 4, 69-79. doi:10.1116/1.3274061

Langley, G. R., Adcock, I. M., Busquet, F. et al. (2017). Towards a $21^{\text {st }}$-century roadmap for biomedical research and drug discovery: Consensus report and recommendations. Drug Discov Today 22, 327-339. doi:10.1016/j.drudis.2016.10.011

Li, G., Lee, C., Agrahari, V. et al. (2019). In vivo measurement of trabecular meshwork stiffness in a corticosteroid-induced ocular hypertensive mouse model. Proc Natl Acad Sci U S A 116, 1714-1722. doi:10.1073/pnas.1814889116

Lv, X., Liu, S. and Hu, Z.-W. (2017). Autophagy-inducing natural compounds: A treasure resource for developing therapeutics against tissue fibrosis. J Asian Nat Prod Res 19, 101-108. doi:10.1080/10286020.2017.1279151

Micheal, S., Yousaf, S., Khan, M. I. et al. (2013). Polymorphisms in matrix metalloproteinases MMP1 and MMP9 are associated with primary open-angle and angle closure glaucoma in a Pakistani population. Mol Vis 19, 441-447.

Osmond, M., Bernier, S. M., Pantcheva, M. B. et al. (2017). Collagen and collagen-chondroitin sulfate scaffolds with uniaxially aligned pores for the biomimetic, three dimensional culture of trabecular meshwork cells. Biotechnol Bioeng 114, 915923. doi:10.1002/bit.26206

Poehlmann, A., Reissig, K., Schönfeld, P. et al. (2013). Repeated $\mathrm{H}_{2} \mathrm{O}_{2}$ exposure drives cell cycle progression in an in vitro model of ulcerative colitis. J Cell Mol Med 17, 1619-1631. doi: $10.1111 / \mathrm{jcmm} .12150$

Pulliero, A., Seydel, A., Camoirano, A. et al. (2014). Oxidative damage and autophagy in the human trabecular meshwork as related with ageing. PLoS One 9, e98106. doi:10.1371/journal. pone. 0098106

Saccà, S. C. and Izzotti, A. (2014). Focus on molecular events in the anterior chamber leading to glaucoma. Cell Mol Life Sci 71,2197-2218. doi:10.1007/s00018-013-1493-z

Saccà, S. C., Gandolfi, S., Bagnis, A. et al. (2016a). From DNA damage to functional changes of the trabecular meshwork in aging and glaucoma. Ageing Res Rev 29, 26-41. doi:10.1016/j. arr.2016.05.012

Saccà, S. C., Gandolfi, S., Bagnis, A. et al. (2016b). The outflow pathway: A tissue with morphological and functional unity. $J$ Cell Physiol 231, 1876-1893. doi:10.1002/jcp.25305

Saccà, S. C., Corazza, P., Gandolfi, S. et al. (2019). Substances of interest that support glaucoma therapy. Nutrients 11, 239. doi:10.3390/nu11020239

Singh, D., Srivastava, S. K., Chaudhuri, T. K. et al. (2015). Multifaceted role of matrix metalloproteinases (MMPs). Front Mol Biosci 2, 19. doi:10.3389/fmolb.2015.00019 
Stapulionis, R., Kolli, S. and Deutscher, M. P. (1997). Efficient mammalian protein synthesis requires an intact F-actin system. J Biol Chem 272, 24980-24986. doi:10.1074/jbc.272.40.24980

Struebing, F. L. and Geisert, E. E. (2015). What animal models can tell us about glaucoma. Prog Mol Biol Transl Sci 134, 365380. doi:10.1016/bs.pmbts.2015.06.003

Taurone, S., Ripandelli, G., Pacella, E. et al. (2015). Potential regulatory molecules in the human trabecular meshwork of patients with glaucoma: Immunohistochemical profile of a number of inflammatory cytokines. Mol Med Rep 11, 1384-1390. doi:10.3892/mmr.2014.2772

Tham, Y.-C., Li, X., Wong, T. Y. et al. (2014). Global prevalence of glaucoma and projections of glaucoma burden through 2040: A systematic review and meta-analysis. Ophthalmology 121, 2081-2090. doi:10.1016/j.ophtha.2014.05.013

Ucciferri, N., Sbrana, T. and Ahluwalia, A. (2014). Allometric scaling and cell ratios in multi-organ in vitro models of human metabolism. Front Bioeng Biotechnol 2, 74. doi:10.3389/ fbioe.2014.00074

Vanamee, É. S. and Faustman, D. L. (2017). TNFR2: A novel target for cancer immunotherapy. Trends Mol Med 23, 10371046. doi:10.1016/j.molmed.2017.09.007

Vandesompele, J., De Preter, K., Pattyn, F. et al. (2002). Accurate normalization of real-time quantitative RT-PCR data by geometric averaging of multiple internal control genes. Genome Biol 3, research0034.1. doi:10.1186/gb-2002-3-7research0034

Vernazza, S., Tirendi, S., Scarfî, S. et al. (2019). 2D- and 3D-cultures of human trabecular meshwork cells: A preliminary assessment of an in vitro model for glaucoma study. PLoS One 14, e0221942. doi:10.1371/journal.pone.0221942

Waduthanthri, K. D., He, Y., Montemagno, C. et al. (2019). An injectable peptide hydrogel for reconstruction of the human trabecular meshwork. Acta Biomater 100, 244-254. doi:10.1016/j.actbio.2019.09.032

Wang, J., Harris, A., Prendes, M. A. et al. (2017). Targeting transforming growth factor- $\beta$ signaling in primary openangle glaucoma. J Glaucoma 26, 390-395. doi:10.1097/ IJG.0000000000000627

Wang, N., Chintala, S. K., Fini, M. E. et al. (2001). Activation of a tissue-specific stress response in the aqueous outflow pathway of the eye defines the glaucoma disease phenotype. Nat Med 7, 304-309. doi:10.1038/85446

Xiang, Y., Li, B., Li, G.-G. et al. (2010). Effects of endothelin-1 on the cytoskeleton protein F-actin of human trabecular meshwork cells in vitro. Int $J$ Ophthalmol 3, 61-63. doi:10.398 0/j.issn.2222-3959.2010.01.14

Zahir, N. and Weaver, V. M. (2004). Death in the third dimension: Apoptosis regulation and tissue architecture. Curr Opin Genet Dev 14, 71-80. doi:10.1016/j.gde.2003.12.005

Zhao, J., Wang, S., Zhong, W. et al. (2016). Oxidative stress in the trabecular meshwork (Review). Int J Mol Med 38, 9951002. doi:10.3892/ijmm.2016.2714

\section{Conflict of interest}

The authors declare that they have no conflicts of interest.

\section{Acknowledgments}

Dr Francesco Oddone and Dr Stefania Vernazza were supported by the Italian Ministry of Health and by Fondazione Roma, Rome, Italy. This work was funded by the award "Omikron Italia 2017 - Marco Centofanti Neuroprotection and Glaucoma”, Omikron srl, Rome, Italy.

We would like to express our gratitude to Ilaria Rizzato, University of Genoa, for revising the English of this paper and to IVTech srl for their technical supporting information. 